\title{
Optimizing Sharpness Measure for Bright Lesion Detection in Retinal Image Analysis
}

\author{
Benson S. Y. Lam ${ }^{1}$, Yongsheng Gao ${ }^{1}$, and Alan Wee-Chung Liew ${ }^{2}$ \\ ${ }^{1}$ Griffith School of Engineering, Griffith University, Australia \\ \{b.lam,yongsheng.gao\}@griffith.edu.au \\ ${ }^{2}$ School of Information and Communication Technology, Griffith University, Australia \\ a.liew@griffith.edu.au
}

\begin{abstract}
Due to the spherical shape nature of retina and the illumination effect, detecting bright lesions in a retinal image is a challenging problem. Existing methods depend heavily on a prior knowledge about lesions, which either a user-defined parameter is employed or a supervised learning technique is adopted to estimate the parameter. In this paper, a novel sharpness measure is proposed, which indicates the degree of sharpness of bright lesions in the whole retinal image. It has a sudden jump at the optimal parameter. A polynomial fitting technique is used to capture this jump. We have tested our method on a public available dataset. Experimental results show that the proposed unsupervised approach is able to detect bright lesions accurately in an unhealthy retinal image and it outperforms existing supervised learning method. Also, the proposed method reports no abnormality for a healthy retinal image.
\end{abstract}

\section{Introduction}

Bright lesions are the symptoms of retinal diseases arising from diabetic retinopathy, hypertensive retinopathy, solar retinopathy, retinal vein or artery occlusion, etc. [1-4]. Retinopathies can progress to blindness or severe loss of vision. However, half of the blindness can be prevented by regular screening and timely treatment [5]. This is a labor intensive process, which requires clinical experts to examine a large number of retinas. Several retina centers such as the Joslin Vision Network and Inoveon Corp. have shown that digital photography is an excellent tool for analyzing retina [6, 7] and computer algorithms are being developed for automatic retinal image analysis $[8,9]$.
The common way to detect the bright lesions is to build a bright lesion map to a retinal image, where each pixel indicates the degree of a pixel belonging to a bright lesion. Existing methods employ one of the following three methods to build the lesion map: contrast normalization, spherical shape linearization and their combinations. Then, a thresholding technique, either defined by user or obtained by supervised learning method, is applied to the bright lesion map and locate bright lesions. For the contrast normalization technique, Osareh et al. [10] applied the local contrast enhancement to a retinal image so to standardize the contrast. Then, a supervised learning is applied to locate the bright lesions. Sánchez et al. [11] combined color normalization and contrast enhancement technique to build the bright lesion map. Then, the Fisher's linear discriminant is applied to locate the bright lesion. As a retina has a spherical shape, bright lesion detection can be influenced negatively and a linearization procedure is applied as a preprocessing step. Walter et al. [12] proposed an unsupervised approach to detect bright lesions by approximating the spherical shape of a retina using morphological operations. The bright lesions are then located by applying thresholding to the difference between the approximated spherical shape image and the original retinal image. Huiqi et al. [13] divided the image into sub-images and then performed linearization. Seed region growing is applied to the pixels having a sharp intensity change. Prasad et al. [14] used the morphological operations to detect bright lesions candidates. Then, the image intensity and edge strength are used to obtain the bright lesion map by a linear classifier. Several researchers use the combination of contrast normalization and spherical shape linearization to build the bright lesion map. Niemeijer et al. [15] adopted several statistical classifiers to build the bright lesions map based on 
many features obtained by applying different kinds of contrast normalizations and spherical shape linearizations to the retinal image. Wang et al. [16] adopted brightness adjustment to a retinal image. Then, a nonlinear thresholding technique approximating the spherical shape of the retina is applied to locate the bright lesions.

Although these methods have an excellent performance, they rely on the thresholding technique in the last step. The ground truth label may not be available for a given dataset while fine-tuning the parameter manually is a tedious task. In this paper, we propose a novel method to determine the optimal threshold in bright lesion map, which optimizes our defined sharpness measure. The sharpness measure indicates the degree of sharpness of bright lesions in the whole retinal image. We also develop a new biased spherical shape linearization. Experimental results show that our proposed sharpness measure is able to determine the threshold accurately.

The organization of this paper is given as following. In Section 2, bright lesions are briefly introduced and the proposed method is explained. Then, experimental results are given in Section 3. Finally, conclusions are given in Section 4.

\section{Proposed Method}

In this section, we first give a brief introduction to bright lesions. Then, our proposed a biased spherical shape linearization and sharpness measure are explained.

\subsection{Bright Lesions}

Bright lesions have a very high intensity in a retina image and the three most important clinical types of bright lesions are: hard exudates, cotton-wool spots and drusen [15]. Figure 1 illustrates these three types of bright lesions [15]. Hard exudates are small white or yellowish deposits with sharp margins. Often, they appear waxy, shiny, or glistening. In Figure 1, the exudates are located near the macula. Cotton wool spots appear as puffy white patches on the retina. The three white patches in the figure are the cotton-wools spot. Drusen are tiny yellow or white accumulations having an irregular flame shape and they are around the macula in the figure.
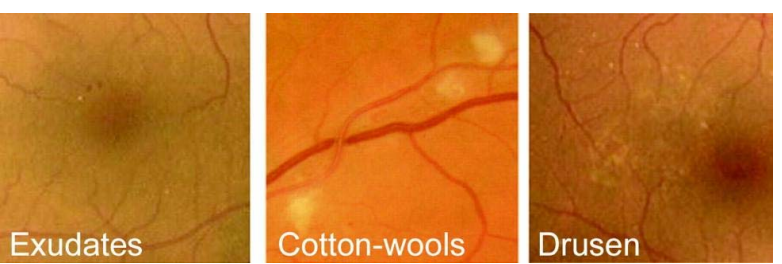

Figure 1. Illustration of exudates, cotton-wools and drusen.

These lesions have a much larger intensity than their neighborhoods. However, due to the spherical shape nature of a retina, some components of a retina can have even a larger intensity than those lesions and a simple thresholding technique cannot detect bright lesions accurately. Figure 2 is an example. Figure 2(a) is a retinal image having bright lesions. The one at the bottom of the image is a cotton-wool spot. After applying a simple thresholding technique (intensity value greater than 100) to the green channel of this image, we obtain a binary image in Figure 2(b), which are given as black pixels. Other than the cotton-wool spot, there is a wrongly detected group in the upper part of the retina. Owing to the illumination effect to the spherical shape of the retina, the high intensity pixels biased towards the upper part of the image. In the next sub-section, a new biased spherical shape linearization is developed to handle this problem.

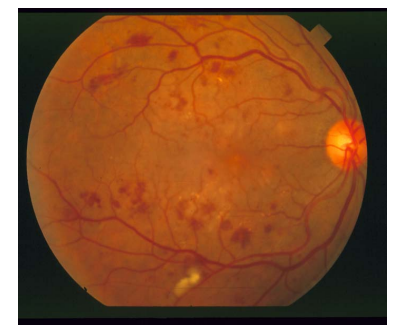

(a)

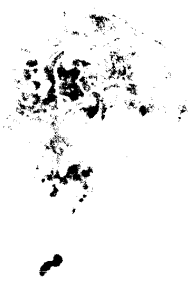

(b)
Figure 2. The influence of the spherical shape nature of a retina and illumination. (a) A retinal image with bright lesions. (b) Applying thresholding to Figure 2(a).

\subsection{A Biased Spherical Shape Linearization}

Retina is a combination of the components: a spherical shaped background retina, vessels, bright lesions, etc. The spherical shaped background retina is the smoothest among the others. The blood vessels are elongated concave structure and its cross section is like a parabola. Bright lesions are white or yellowish patches. They have a steep intensity transition property and are non-differentiable along their boundaries. Thus, if a pixel belongs to a vessel, it can be thought of as a linear combination of background retina minus a 
parabola shaped (due to its concave property). If a pixel belongs to bright lesion, it is a combination of background retina and a non-differentiable function. This structure is like a Fourier series

$$
f(x)=\frac{a_{0}}{2}+\sum_{n=1}^{\infty}\left[a_{n} \cos (n x)+b_{n} \sin (n x)\right]
$$

where $a_{\mathrm{n}}$ and $b_{\mathrm{n}}$ are the Fourier coefficients. $n$ here represents the frequency of the signal. A smaller $n$ represents a lower frequency and thus smoother. If the function at a point $x=x_{0}$ only needs small frequency sine and cosine functions for the approximation, it must be very smooth. Otherwise, $f\left(x_{0}\right)$ is not smooth.

This is similar to the retinal structure. The low frequency structure can refer to as the background retina while the high frequency structure can refer to the vessels and bright lesions. Moreover, the illumination effect just biased the peak of the spherical shape towards a specific direction. It does not create non-smoothness and must also be smooth. Thus, the low frequency structure can capture the effect. In order to remove the background retina, we have to eliminate the low frequency structure.

Our strategy to eliminate the low frequency components is by Fourier transform. For a given 2D dataset $\left\{x_{m, n}: m=0, \ldots, M ; n=0, \ldots, N\right\}$, its Fourier transform is given by

$$
X_{k, l}=\frac{1}{\sqrt{M N}} \sum_{m=0}^{M} \sum_{n=0}^{N-1} x_{m, n} \exp \left\{-\left(\frac{i 2 \pi k m}{M}+\frac{i 2 \pi l n}{N}\right)\right\}
$$

for $k=0, \ldots, M ; l=0, \ldots, N . x_{\mathrm{m}, \mathrm{n}}$ is the $(m, n)$ th pixel of the retinal image. Then, the high frequency components are discarded and only low frequency components are remained. In this paper, we only retain the first two components and we obtain the approximated biased spherical shape $b_{k, l}$,

$$
b_{k, l}=\frac{1}{\sqrt{M N}} \sum_{m=0}^{2} \sum_{n=0}^{2} X_{m, n} \exp \left\{\left(\frac{i 2 \pi k m}{M}+\frac{i 2 \pi l n}{N}\right)\right\}
$$

for $k=0, \ldots, M ; l=0, \ldots, N$. Then, the normalized image $w_{\mathrm{k}, 1}$ is given by

$$
w_{k, l}=x_{k, l}-b_{k, l}
$$

Figures 3(a) and (b) show the approximated biased spherical shape and its contour plot of the retinal image given in Figure 2(a). The numbers on each contour lines in Figure 3(b) indicate the corresponding intensity levels in Figure 3(a). The contour plot shows that the highest value is shifted a little bit upwards. This agrees with the shifted high intensity of the retinal image.

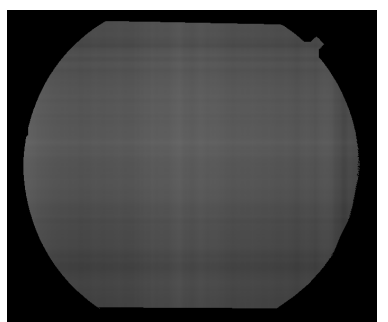

(a)

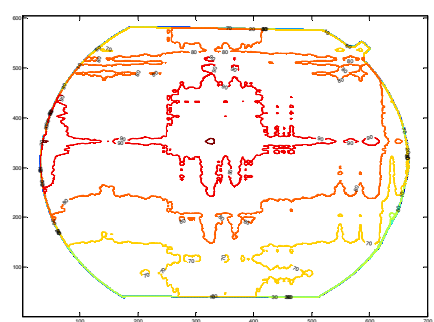

(b)

Figure 3. Retinal biased spherical shape approximation. (a) The approximated biased spherical shape. (b) The contour plot of (a).

\subsection{Sharpness Measure}

Although the linearization can eliminate the biased spherical shape effect, noise can still be present and surrounding the bright lesions. In this section, a sharpness measure is proposed, which indicates the degree of sharpness of the bright lesion in the whole retinal image.

To measure the sharp intensity change, a thresholding mapping is developed. For $t$ given thresholds $h(i)$ (for $i=0, \ldots t$ ), the normalized image $\mathbf{w}_{k, l}$ is mapped to a threshold space by the following equation:

$$
m(i)=\frac{1}{\operatorname{card}\left(w_{k, l}>h(i)\right)} \sum_{\substack{k, l \\ w_{k, l}>h(i)}} w_{k, l}
$$

where $\operatorname{card}(\bullet)$ is the cardinality. That is, $m(i)$ is the means of the $\mathrm{w}_{k, l}$ which are greater than the threshold $h(i)$. If a threshold $h\left(i_{*}\right)$ is above the noise and reaches the bottom of the lesions, there must be a sudden jump from $m\left(i_{*}-1\right)$ to $m(i *)$. The reason is given as below. If $i<i_{*}$, the average is taken among the mixture of the signal and noise. If $i>i_{*}$, the average is taken among the signal only. As the bright lesions always have a sharp intensity change, this creates the sudden jump. This is further illustrated by the following example. Figure 4(a) is a simulated bright lesion with noise at the two ends. Figure 4(b) is the threshold mapping in the range $[0.9,1.2]$. The $\mathrm{x}$-axis represents the threshold $h(i)$ while the y-axis represents the threshold mapping $m(i)$. We can see that there is a sudden jump in between [0.9 1.0], which is the upper bound of the noisy signal in Figure 4(a). Thus, the threshold level $i$ causes the sudden jump is the optimal threshold $\mu$. After applying the method given in Table 1, the threshold $\mu$ is approximated as 0.9615 .

The sudden jump creates a locally convex property along the threshold mapping curve, which the second order derivative of the curve in Figure 4(b) must be negative [17]. This locally convex property can be 
captured by curve fitting for a segment in a narrower threshold range. For a given range of threshold level $h(i)$ for $i=r_{1}, \ldots, r_{2}\left(0<r_{1}, r_{2}<t\right)$, we consider the following regression problem:

$$
m(i)=\beta_{0}+\beta_{1} h(i)+\beta_{2} h^{2}(i)
$$

where $\beta_{0}, \beta_{1}$ and $\beta_{2}$ are the unknowns. In this polynomial, the second order derivative is $\beta_{2}$, which must be negative. Thus, the threshold having the largest negative value of $\beta_{2}$ is the optimal threshold $\mu$. However, the noisy signal can cause convergence to local optimal. In our case, we reduce the number of unknowns by approximating the first order derivative of $y(i)$. A smaller number of unknown variables can lower the change of being convergence to local optimal. The first order polynomial is then given by:

$$
m^{\prime}(i)=\beta_{1}+\beta_{2} h(i)
$$

$m^{\prime}(i)$ is approximated by the difference between the averages of the normalized intensity outside and inside the boundary of $\mathbf{w}>h(i)$. Figures 5(a) and (b) give an example of inward and outward boundaries. The detailed implementation is given in Table 1.

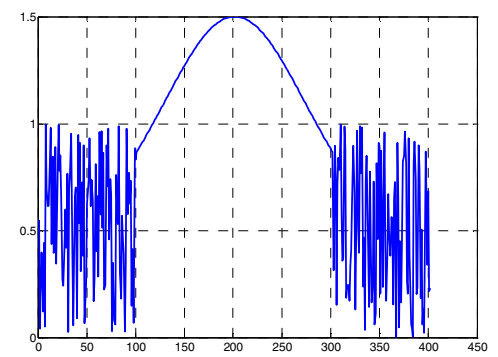

(a)

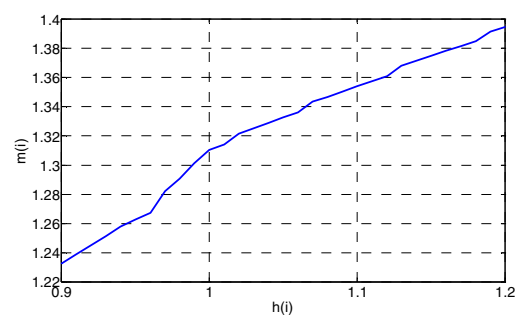

(b)

Figure 4. An illustration of the threshold mapping. (a) A simulated bright lesion. (b) The threshold mapping in the range $[0.9,1.2]$.

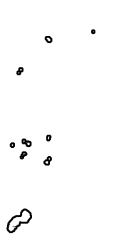

(b) (c)

Figure 5. The boundaries of $\mathbf{w}>\mathrm{h}(i)$ of the retinal image given in Figure 2(a). (a) A plane of $\mathbf{w}>\mathrm{h}(i)$. (b) Inward boundary of (a). (c) Outward boundary (a).

\section{Table 1. Bright Lesion Detection.}

Step 0: Denote the maximum feature value $\mathbf{m}_{\mathrm{w}}$ of the normalized image $\mathbf{w}$.

Step 1: Divide the interval $\left[0, \mathbf{m}_{\mathrm{w}}\right]$ uniformly into $t$ levels and denote each level as $\mathrm{h}(i)$.

For the $i$ th level, do

Step 2: Compute the average normalized intensity $I_{\text {out }}$ in an a outward binary plane, whose pixel is one if it is $p$ pixels in the outward normal direction away from the boundary of $\mathbf{w}>\mathrm{h}(i)$.

Step 3: Compute the average normalized intensity $I_{\text {in }}$ in an a inward binary plane, whose pixel is one if it is $p$ pixels in the inward normal direction away from the boundary of $\mathbf{w}>\mathrm{h}(i)$.

Step 4: $g(i)=\left(I_{\text {out }}-I_{\text {in }}\right) / 2$.

Step 5: Fit the linear regression polynomial:

$g(i)=\beta_{1}+\beta_{2} h(i)$

and record $\beta(i)=\beta_{2}$.

Step 6: if $\beta(i)$ is negative and $|\beta(i)|>|\beta(j)|$ for $\forall j<i$, then, output the $h(i)$ as the optimal threshold and stop. Otherwise, continue.

End do

As the bright lesions must be above the spherical shaped background retina, it must belong to the positive part of the normalized image w. The threshold interval $\left[0, \mathbf{m}_{\mathrm{w}}\right]$ is then started from zero. In this paper, the total number of levels $t$ is set as 50 and the pixel $p$ is taken as 5. The polynomial fitting range $r_{1}$ and $r_{2}$ are set as $r_{1}=i-5$ and $r_{2}=i+5$. Figure 5(a) gives the detection result using the proposed method to the retinal image given in Figure 2(a). Figure 6 shows the 
upper and middle parts of the retinal image and the corresponding detection results.

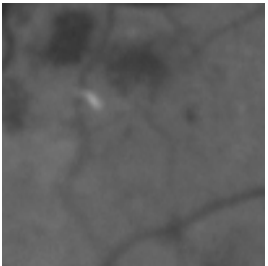

(a)

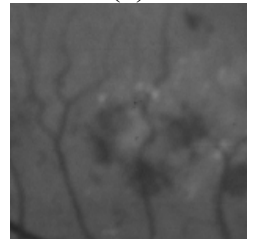

(c)

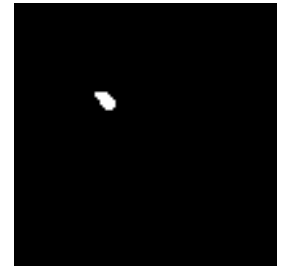

(b)

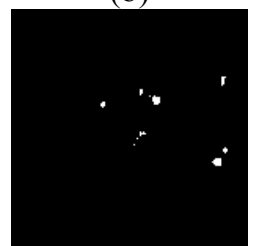

(d)
Figure 6. Detection result using the proposed method. (a) The upper part of the retina in Figure 2(a). (b) The upper part of the detected result. (c) The middle part of the retina in Figure 2(a). (d) The middle part of the detected result.

\section{Experimental Results}

The performance of the proposed method is evaluated on a publicly available STARE database. The STARE ${ }^{1}$ database contains 20 images with 10 pathological and 10 normal retinal images. Each of the images has a size of $700 \times 605$ pixels with 8 bits per color channel. The green channel of a retinal image is used as input because it contains the richest information [12]. We have also implemented the method of Wang et al. [16] for comparison. As their method is a supervised learning method, we have manually label the bright lesions in the image as ground truth for the classifier training. Also, we use the same set of parameters to detect the bright lesion in the bright lesion maps.

Figure 7(a) shows a retinal image having lots of bright lesions in the upper right hand side. Figure 7(b) shows the detection result using the method of Wang et al.. The sizes of the detected bright lesions are much bigger than the real one in Figure 7(a). Moreover, there are two lines wrongly detected at the left hand bottom in the image. This can be caused by the strong contrast along the vessels. Figure 7(c) is the detection result using the proposed method. We can see that the sizes of the detected bright lesions are almost the same as the one in Figure 7(a). Moreover, the two lines in Figure 7(b) do not appear.

\footnotetext{
${ }^{1}$ http://www.parl.clemson.edu/stare/probing/
}

Figure 8(a) shows a healthy retinal image without any lesion. The detection result using the method of Wang et al. is given in Figure 8(b). Again, due to the high contrast along the vessels in the bottom of the image, the method wrongly detects the region as bright lesions. Figure 8(c) shows the result using the proposed method. We can see that only the optic disc is detected and no other bright lesions are found, which can be eliminated by many existing optic disc detection algorithms. In the experiments given in Figures 7 and 8, we found that it is not trivial the find a same set of parameter to perform well in both retinal images. Our proposed method yielded two different threshold for the two retinal images by optimizing the sharpness measure.

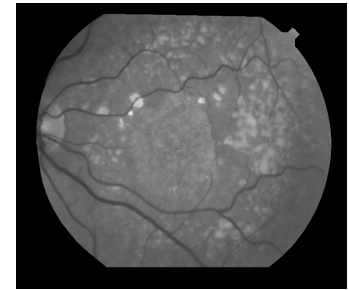

(a)

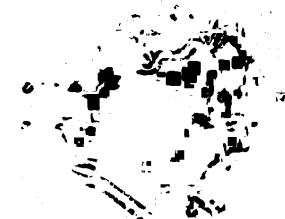

(b)

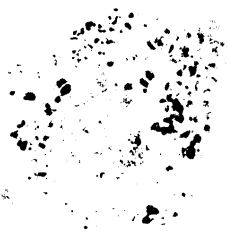

(c)

Figure 7. Performance comparisons on an unhealthy retinal image. (a) A retinal image having lots of bright lesions. (b) Detection result using the method of Wang et al. (c) Detection result using proposed method. 


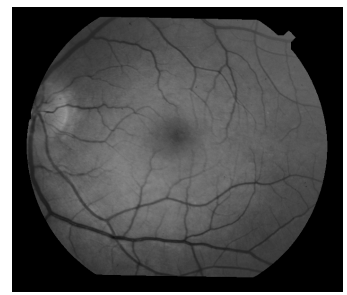

(a)

(c)

Figure 8. Performance comparisons on a healthy retinal image. (a) A retinal image without any bright lesions.

(b) Detection result using the method of Wang et al.

(c) Detection result using proposed method.

\section{Conclusions}

Selecting a suitable parameter for bright lesion map for bright lesion detection is a challenging problem. In this paper, a novel sharpness measure is proposed and its sudden jump property provides an important clue for optimal parameter selection. We have also proposed a new biased spherical shape linearization based on Fourier transform. Experimental results show that the proposed unsupervised approach is able to detect bright lesions accurately in an unhealthy retina and it outperforms existing supervised learning method. More importantly, the proposed method reports no abnormality for a healthy retinal image.

\section{References}

[1] The Eye Diseases Prevalence Research Group, "Prevalence of Age Related Macular Degeneration in the United States," Arch. Ophth., Vol. 122, pp. 564 $572,2004$.

[2] The Eye Diseases Prevalence Research Group, "The Prevalence of Diabetic Retinopathy Among Adults in the United States," Arch. Ophth., Vol. 122, no. 4, pp. 552-563, 2004.

[3] The Eye Diseases Prevalence Research Group, "Prevalence of Open Angle Glaucoma Among Adults in the United States," Arch. Ophth., Vol. 122, no. 4, pp. 532-538, 2004.

[4] Springhouse, Professional Guide to Diseases, Lippincott Williams \& Wilkins; Seventh Edition edition, 2001.
[5] J. A. Shoemaker, Vision problems in the U.S. Tech. Rep. U.S. National Institute of Health, 2002.

[6] A. A. Cavallerano, J. D. Cavallerano, P. Katalinic, A. M. Tolson, and L. P. Aiello, "Use of Joslin Vision Network Digital-video Nonmydriatic Retinal Imaging to Assess Diabetic Retinopathy in a Clinical Program," Retina, vol. 23, pp. 215-223, April 2003.

[7] S. R. Fransen, T. C. Leonard-Martin, W. J. Feuer, and P. L. Hildebrand, "Clinical Evaluation of Patients with Diabetic Retinopathy: Accuracy of the Inoveon Diabetic Retinopathy-3DT System," Ophthalmology, Vol. 109, pp. 595-601, Mar. 2002.

[8] M. Niemeijer, B. van Ginneken, J. Staal, M. SuttorpSchulten and M. Abramoff, "Automatic Detection of Red Lesions in Digital Color Fundus Photographs," IEEE Transactions on Medical Imaging, Vol. 24, pp. 584-592, 2005.

[9] N. Patton, T. Aslam, T. MacGillivray, I. Deary, B. Dhillon, R. Eikelboom, K. Yogesan and I. Constable, "Retinal Image Analysis: Concepts, Applications and Potential," Progress in Retinal and Eye Research, Vol. 25, pp. 99-127, 2006.

[10] A. Osareh, M. Mirmehdi, B. Thomas and R. Markham, "Automated identification of diabetic retinal exudates in digital colour images", British Journal of Ophthalmology, Vol. 87, pp.1220-1223, 2003.

[11] C. Sánchez, R. Hornero, M. López, M. Aboy, J. Poza, D. Abásolo, "A novel automatic image processing algorithm for detection of hard exudates based on retinal image analysis", Medical Engineering \& Physics, Vol. 3, pp. 350-357, 2008.

[12] T. Walter, J. Klein, P. Massin, A. Erginay, "A Contribution of Image Processing to the Diagnosis of Diabetic Retinopathy-Detection of Exudates in Color Fundus Images of the Human Retina", IEEE Transactions on Medical Imaging, Vol. 21, pp. 12361243, 2002.

[13] L. Huiqi, O. Chutatape, "Automated Feature Extraction in Color Retinal Images by a Model Based Approach", IEEE Transactions on Biomedical Engineering, Vol. 51, pp. 246 - 254, 2004.

[14] S. Prasad, A. Jain and A. Mittal. "Automated Feature Extraction for Early Detection of Diabetic Retinopathy in Fundus Images," IEEE Conference on Computer Vision and Pattern Recognition, to appear, 2009.

[15] M. Niemeijer, B. Ginneken, S. Russell, M. SuttorpSchulten and M. Abràmoff, "Automated Detection and Differentiation of Drusen, Exudates, and Cotton-Wool Spots in Digital Color Fundus Photographs for Diabetic Retinopathy Diagnosis", Investigative Ophthalmology and Visual Science, Vol. 48, pp. 2260-2267, 2007.

[16] H. Wang, W. Hsu, K. Goh and M. Lee, "An Effective Approach to Detect Lesions in Color Retinal Images", IEEE Conference on Computer Vision and Pattern Recognition, Vol, 2, pp. 181-186, 2000.

[17] W. Kaplan, Advanced calculus, 4th edition, Publisher Reading, Mass.: Addison-Wesley, 1991. 